\title{
Peripheral Blood Vessel
}

National Cancer Institute

\section{Source}

National Cancer Institute. Peripheral Blood Vessel. NCI Thesaurus. Code C83001.

A blood vessel located in the following sites: upper extremities, lower extremities, lower abdomen, head, or neck. 